journal club

\title{
Signifikante Symptomverbesserung
}

Fragestellung: Wie wirksam, sicher und verträglich ist Brexpiprazol bei der Behandlung der akuten Schizophrenie?

Hintergrund: Moderne Antipsychotika wirken überwiegend nur auf die Positivsymptomatik der Schizophrenie und sind, je nach pharmakologischem Profil, mit unterschiedlichsten Nebenwirkungen behaftet, die die Medikamentenadhärenz beeinträchtigen können. Längerfristige Medikamentenadhärenz ist jedoch eine wesentliche Grundlage der Rückfallprävention.

Brexpiprazol ist ein Modulater der Serotonin-Dopamin-Aktivität, der als partieller Agonist am Serotonin 5-HT1A- und Dopamin-D2-Rezeptor und am 5-HT2A- und Noradrenalin alpha1B/2C-Rezeptor hingegen antagonistisch wirkt. Im Vergleich zum bisher einzigen verfügbaren partiellen D2-Agonisten Aripiprazol zeigt Brexpiprazol eine geringere intrinsische Aktivität am D2-Rezeptor, was ein geringeres Potenzial für unerwünschte Nebenwirkungen vermuten lässt. Durch sein ausgewogenes Rezeptorbindungsprofil ist die Gefahr einer übermäßigen Antriebssteigerung und Induktion motorischer Nebenwirkungen wohl gering. Aufgrund seiner nur moderaten Affinität zum H1-Rezeptor sind darüber hinaus auch allenfalls geringfügige sedierende Effekte zu erwarten.

Correll CU, Skuban A, Ouyang J et al. Efficacy and safety of brexpiprazole for the treatment of acute Schizophrenia: A 6-week randomized, double-blind, placebo-controlled trial. Am J Psychiatry 2015; 172: 870-80
Patienten und Methodik: Die multizentrische, randomisierte, placebokontrollierte Doppelblindstudie schloss 636 Patienten zwischen 18 und 65 Jahren mit akut exazerbierter Schizophrenie ein. Psychiatrische Komorbidität stellte ein Ausschlusskriterium dar. Die Patienten erhielten randomisiert Brexpiprazol in einer Dosierung von 0,25 mg, $2 \mathrm{mg}$ oder 4 mg oder Placebo. Primärer Outcome war die Veränderung des PANSS-Gesamtscores von Baseline bis sechs Wochen nach Therapiebeginn, hauptsächlicher sekundärer Outcome der Schweregrad der Clinical Global Imperession Scale (CGI).

Ergebnisse: Zum Baselinezeitpunkt betrugen der mittlere PANSS-Gesamtscore 95,2 und der mittlere CGI-Schweregrad 4,9 Die Studie wurde von 62,2\%, 68,1\%, und 67,2\% der Patienten in der 0,25-, 2- und 4-mg-Brexpiprazolgruppe gegenüber 59,2\% in der Placebogruppe abgeschlossen. Nach sechs Wochen zeigte sich in der Gruppe, die 2 mg oder 4 mg Brexpiprazol erhielt, eine signifikant größere Reduktion des PANSS-Gesamtscores (- 8,72 bzw. - 7,64) und des CGI-Schweregrades $(-0,33$ bzw. - 0,38) gegenüber Placebo. Die Number Needed to Treat betrug 6 bis 8 . Auch in den PANSS-Subskalen für Positiv- und Negativsymptomatik fand sich im Therapieverlauf eine signifikante Abnahme unter 2 beziehungsweise $4 \mathrm{mg}$ Brexpiprazol gegenüber Placebo (Positiv: -2.22 bzw. -2.44 , Negativ: $-1,78$ bzw. $-1,41$ ). Die soziale Funktionsfähigkeit, gemessen mit der Personal and Social Performance Scale, besserte sich lediglich unter $2 \mathrm{mg}$ signifikant $(2,89 ; \mathrm{p}=0,03)$. Unter Brexpiprazol war Akathisie die häufigste Nebenwirkung (2 mg: 4,4\%; 4 mg: 7,2\%; Placebo: 2,2\%). Zudem zeigte sich eine moderate Gewichtszunahme $(1,45$ und $1,28 \mathrm{~kg}$ unter 2 bzw. 4 mg Brexpiprazol, 0,42 kg unter Placebo).

Schlussfolgerungen: Brexpiprazol zeigte in Dosierungen von $2 \mathrm{mg}$ und $4 \mathrm{mg}$ pro Tag gegenüber Placebo eine signifikante Symptomverbesserung bei guter Verträglichkeit bei Patienten mit akuter Exazerbation einer Schizophrenie.

\section{- Kommentar von Irina Falkenberg, Marburg}

\section{Neue Therapieoption für die Schizophrenie?}

Der Bedarf für neue medikamentöse Optionen in der Schizophreniebehandlung ist immens. Unerwünschte Nebenwirkungen der gängigen Antipsychotika sind wesentliche Gründe für selbstständiges Absetzen der Medikamente durch die Patienten und somit für das Auftreten von Rezidiven. Die großangelegte Studie mit gutem Design (JADAD-Score 5) erbringt Hinweise auf eine gut wirksame und gute verträgliche Erweiterung des Repertoires antipsychotischer Behandlungsoptionen. Insbesondere die geringen metabolischen Nebenwirkungen von Brexpiprazol heben die Substanz von anderen etablierten Antipsychotika ab. Inwieweit dieses vielversprechende Profil auch längerfristig mit einer erhöhten Medikamentencompliance einhergehen wird, einem Faktor, den die Autoren als wesentliche Motivation für die Studie benannten, bleibt abzuwarten. Die Abbruchraten lagen nach sechs Wochen Behandlung mit Brexpiprazol zwischen $30 \%$ und $40 \%$. Längere Beobachtungsszeiträume sind hier notwendig, um Fakto- ren, die zum Behandlungsabbruch beitragen evaluieren zu können. Auch der Vergleich von Brexpiprazol mit aktiven Substanzen sowie Untersuchungen zu seiner Wirksamkeit unter realitätsnäheren klinischen Bedingungen (d.h. bei Patienten mit komorbiden Störungen) stehen noch aus.

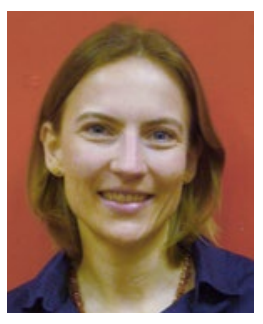

Dr. med. Irina Falkenberg, Marburg

Klinik für Psychiatrie und Psychotherapie Philipps-Universität Marburg - UKGMz E-Mail: irina.falkenberg@med.uni-marburg.de 